\title{
The existence of an inverse limit of inverse system of measure spaces - a purely measurable case*
}

\author{
Miklós Pintér \\ Corvinus University of Budapest ${ }^{\dagger}$
}

July 26, 2009

\begin{abstract}
The existence of an inverse limit of an inverse system of (probability) measure spaces has been investigated since the very beginning of the birth of the modern probability theory. Results from Kolmogorov [10], Bochner 22, Choksi [5], Metivier [14, Bourbaki [3] among others have paved the way of the deep understanding of the problem under consideration. All the above results, however, call for some topological concepts, or at least ones which are closely related topological ones. In this paper we investigate purely measurable inverse systems of (probability) measure spaces, and give a sufficient condition for the existence of a unique inverse limit. An example for the considered purely measurable inverse systems of (probability) measure spaces is also given.
\end{abstract}

\section{Introduction}

The existence of an inverse limit of an inverse system of (probability) measure spaces is an important question in probability theory (see e.g. Kolmogorov [10]), in the theory of stochastic processes (see e.g. Rao [18]), and in some sense surprisingly in game theory.

*The author thanks the Hungarian Scientific Research Fund (OTKA) and the János Bolyai Research Scholarship of the Hungarian Academy of Sciences for financial support.

${ }^{\dagger}$ Department of Mathematics, Corvinus University of Budapest, 1093 Hungary, Budapest, Fővám tér 13-15., miklos.pinter@uni-corvinus.hu 
On the field of game theory, actually on the subfield of games with incomplete information, the investigation of the so called hierarchies of beliefs - in the language of mathematics: inverse systems of (probability) measure spaces, see Harsányi [7], Mertens and Zamir [12], Brandenburger and Dekel [4], Heifetz [8], Mertens at al. [13], Pintér [17] among others - calls for the applications of certain existence results.

All the previous results - Kolmogorov, Bochner [2], Choksi [5], Metivier [14], Bourbaki [3] among others - on the existence of an inverse limit of an inverse system of measure spaces, however, calls for topological concepts, various compactness concepts, even if it does not seems at first glance (see e.g. Metivier's result).

The case of hierarchies of beliefs is, however, a special one, it is quite natural to consider these inverse systems of measure spaces as purely measurable ones (see e.g. Heifetz and Samet [9]), so in order to examine the existence of an inverse limit of an inverse system of measure spaces in the purely measurable framework we need a purely measurable existence result.

The main result of this paper is Theorem 7. which provides purely measurable sufficient condition for the existence of a unique inverse limit. Actually, in this paper we introduce the concept of $\varepsilon$-completeness (see Definition 6.), and show that it is sufficient but not necessary condition for the existence of a unique inverse limit of an inverse system of (probability) measure spaces. In our opinion, this result is a common generalization of all the previously cited results (Kolmogorov, Bochner, Choksi, Metivier, Bourbaki), and we discuss it in more detail after Theorem 7 .

The mathematical core of a game theoretical problem, Proposition 11, as an example for a class of $\varepsilon$-complete inverse systems of (probability) measure spaces, is also given.

The setup is as follows. In the next section we provide some basic concepts of inverse systems and inverse limits. In Section 3. we present our main result, and Section 4. is about the above mentioned example for the application of our main result. In the last section we mention some obvious generalizations, and conclude briefly.

\section{Inverse systems, inverse limits}

First some notions and notations. In this paper we work with probability measures, hence if we do not indicate differently we mean every measure is a probability measure.

Let $A$ be an arbitrary set, then $\# A$ is for the cardinality of set $A$. For any $A \subseteq \mathcal{P}(X): \sigma(A)$ is the coarsest $\sigma$-field which contains $A$. Let $(X, \mathcal{M})$ 
and $(Y, \mathcal{N})$ be arbitrary measurable spaces. Then $(X \times Y, \mathcal{M} \otimes \mathcal{N})$ or briefly $X \otimes Y$ is the measurable space on the set $X \times Y$ equipped by the $\sigma$-field $\sigma(\{A \times B \mid A \in \mathcal{M}, B \in \mathcal{N}\})$.

Let $\mathcal{A} \subseteq \mathcal{P}(\mathcal{X})$ be a set ring, $\mathcal{C} \subseteq \mathcal{A}, \mu$ additive set function on $\mathcal{A} ; \mu$ is $\mathcal{C}$-regular if for arbitrary $\epsilon>0$ and for arbitrary $A \in \mathcal{A}, \exists C \in \mathcal{C}$ such that $C \subseteq A$ and $\mu(A \backslash C)<\epsilon$.

Set system $\mathcal{A} \subseteq \mathcal{P}(X)$ is a $\sigma$-compact set system, if for any $\left\{A_{n}\right\} \subseteq \mathcal{A}$ : $\bigcap_{n} A_{n}=\emptyset$ implies that $\exists n^{*}$ s. t. $\bigcup_{n=1}^{n^{*}} A_{n}=\emptyset$.

Let $(X, \mathcal{M}, \mu)$ be an arbitrarily fixed (probability) measure space. Then the set function $\mu^{*}$ on $\mathcal{P}(X)$ is defined as follows: $\forall A \in \mathcal{P}(X): \mu^{*}(A) \stackrel{\circ}{=}$ $\inf _{A \subseteq B \in \mathcal{M}} \mu(B)$ (therefore $\mu^{*}$ is an outer measure).

For any topological space $(X, \tau): B(X, \tau)$ is for the Borel $\sigma$-field.

We say the measurable spaces $(X, \mathcal{M})$ and $(Y, \mathcal{N})$ are measurable isomorphic if there is such a bijection $f$ between them that both $f$ and $f^{-1}$ are measurable.

Next, we discuss the basic concepts of inverse systems and inverse limits.

Definition 1. Let $(I, \leq)$ be a preordered set, $\left(X_{i}\right)_{i \in I}$ be a family of nonvoid sets, and $\forall i, j \in i$ s.t. $i \leq j: f_{i j}: X_{j} \rightarrow X_{i}$. The system $\left(X_{i},(I, \leq), f_{i j}\right)$ is an inverse system if it meets the following points, $\forall i, j, k \in I$ s.t. $i \leq j$ and $j \leq k$ :

1. $f_{i i}=i d_{X_{i}}$,

2. $f_{i k}=f_{i j} \circ f_{j k}$.

The inverse system (projective system) is a system of sets connected in a certain way.

Definition 2. Let $\left(\left(X_{i}, \mathcal{A}_{i}, \mu_{i}\right),(I, \leq), f_{i j}\right)$ be such an inverse system that $\forall i \in I:\left(X_{i}, \mathcal{A}_{i}, \mu_{i}\right)$ is a measure space. The inverse system $\left(\left(X_{i}, \mathcal{A}_{i}, \mu_{i}\right),(I, \leq\right.$ ),$\left.f_{i j}\right)$ is an inverse system of measure spaces if it meets the following points, $\forall i, j \in I$ s.t. $i \leq j$ :

1. $f_{i j}$ is a $\mathcal{A}_{j}$-measurable function,

2. $\mu_{i}=\mu_{j} \circ f_{i j}^{-1}$. 
Definition 3. Let $\left(X_{i},(I, \leq), f_{i j}\right)$ be an inverse system. Let $X \stackrel{\circ}{=} \prod_{i \in I} X_{i}$, and $P \stackrel{\circ}{=}\left\{x \in X \mid p r_{i}(x)=f_{i j} \circ p r_{j}(x), \forall(i \leq j)\right\}$, where $\forall i \in I: p r_{i}$ is the coordinate projection from $X$ to $X_{i}$.

Then $P$ is called the inverse limit of the inverse system $\left(X_{i},(I, \leq), f_{i j}\right)$, and it is denoted by $\lim _{(}\left(X_{i},(I, \leq), f_{i j}\right)$.

Moreover, let $\left.p_{i} \stackrel{\overbrace{}}{=} p r_{i}\right|_{P}$, so $\forall i . j \in I$ s.t. $i \leq j: p_{i}=f_{i j} \circ p_{j}$.

The inverse limit is the generalization of the Cartesian product. If $\leq$ in $(I, \leq)$ is the empty relation, then the inverse limit is the Cartesian product.

Definition 4. Let $\left(\left(X_{i}, \mathcal{A}_{i}, \mu_{i}\right),(I, \leq), f_{i j}\right)$ be an inverse system of measure spaces, and $P \stackrel{\lim }{\leftrightarrows}\left(X_{i},(I, \leq), f_{i j}\right)$. Then the $(P, \mathcal{A}, \mu)$ measure space is the inverse limit of the inverse system of measure spaces $\left(\left(X_{i}, \mathcal{A}_{i}, \mu_{i}\right),(I, \leq), f_{i j}\right)$ and it is denoted by $(P, \mathcal{A}, \mu) \stackrel{\circ}{=} \lim \left(\left(X_{i}, \mathcal{A}_{i}, \mu_{i}\right),(I, \leq),\left.f_{i j}\right|_{i \leq j}\right)$. if it meets the following points, $\forall i \in I$ :

1. $\mathcal{A}$ is the coarsest $\sigma$-algebra w.r.t. $p_{i}$ is $\mathcal{A}$-measurable,

2. $\mu$ is such a measure that $\mu \circ p_{i}^{-1}=\mu_{i}$.

Kolmogorov's [10] extension theorem is an inverse limit result. It states the following: let consider a family of finite dimensional distributions on $\mathbb{R}^{d}$ $(d<\infty)$ s.t. they are consistent, i.e. $\forall n: \mu_{n}$ on $\left(\mathbb{R}^{n}, B\left(\mathbb{R}^{n}\right)\right.$ is the marginal distribution of $\mu_{n+1}$ on $\left(\mathbb{R}^{n+1}, B\left(\mathbb{R}^{n+1}\right)\right)$. Then there exists such a unique distribution $\mu$ on $\left(\mathbb{R}^{\mathbb{N}}, B\left(\mathbb{R}^{\mathbb{N}}\right)\right)$ that $\forall n$ : $\mu_{n}$ is the marginal distribution of $\mu$. This theorem is called the Kolmogorov Extension Theorem. In this case the inverse system of measure spaces is as follows: $\left(\left(\mathbb{R}^{n}, B\left(\mathbb{R}^{n}\right), \mu_{n}\right), \mathbb{N}, p r_{m n}\right)$, where $p r_{m n}: \mathbb{R}^{n} \rightarrow \mathbb{R}^{m}$ is a coordinate projection. Therefore, the Kolmogorov Extension Theorem states that $\lim _{\longleftarrow}\left(\left(\mathbb{R}^{n}, B\left(\mathbb{R}^{n}\right), \mu_{n}\right), \mathbb{N}, p r_{m n}\right)$ exists and is unique.

Since if $\lim _{\longleftarrow}\left(X_{i},(I, \leq), f_{i j}\right) \neq \emptyset$ then $\bigcup_{i} p_{i}^{-1}\left(\mathcal{M}_{i}\right)$ is an algebra (field), and set function $\mu$ defined on $\bigcup_{i} p_{i}^{-1}\left(\mathcal{M}_{i}\right)$ as $\forall i \in I: \mu \circ p_{i}^{-1}=\mu_{i}$ is an additive set function, the main problem of the existence of measure inverse limit is the $\sigma$-additivity of $\mu$.

Numerous results discuss this problem, the most important ones are as follows: Kolmogorov, Bochner [2], Choksi's [5], Millington and Sion [15], Metivier's [14], Mallory and Sion's [11], Bourbaki's [3]. The next theorem is a variant of Metivier's result, which can be considered as the most general one, at least in this framework, among the above result 
Theorem 5 (Metivier). Let $\left(\left(X_{n}, \mathcal{M}_{n}, \mu_{n}\right), \mathbb{N}, f_{m n}\right)$ be an inverse system of (probability) measure space ․ If $\forall m, n \in \mathbb{N}$ s.t. $m \leq n$ :

1. $\mathcal{C}_{n} \subseteq \mathcal{M}_{n}$ is $\sigma$-compact set system,

2. $f_{m n}$ is a surjective (onto) function,

3. $f_{m n}\left(\mathcal{C}_{n}\right) \subseteq \mathcal{C}_{m}$

4. $\forall x_{m} \in X_{m}: f_{m n}^{-1}\left(\left\{x_{m}\right\}\right) \cap \mathcal{C}_{n}$ is $\sigma$-compact set system,

5. $\forall C_{k} \in \mathcal{C}_{n}, k \in \mathbb{N}: \bigcap_{k} C_{k} \in \mathcal{C}_{n}$,

6. $\mu_{n}$ is $\mathcal{C}_{n}$-regular,

then

$$
(X, \mathcal{M}, \mu) \stackrel{\circ}{=} \lim _{\longleftarrow}\left(\left(X_{n}, \mathcal{M}_{n}, \mu_{n}\right), \mathbb{N},\left.f_{m n}\right|_{m \leq n}\right)
$$

exists and is unique, moreover $\mu$ is $\mathcal{C}$-regular, where $\mathcal{C}$ consists of such sets that $\forall C \in \mathcal{C}: \exists C_{n} \in \mathcal{C}_{n}$ s.t. $\forall n: f_{n n+1}^{-1}\left(C_{n}\right) \subseteq C_{n+1}$ and $C=\lim \left(C_{n}, \mathbb{N}, f_{m n}\right)$, and $\mathcal{C}$ is $\sigma$-compact set system.

Proof. See Metivier 3.2. Theoreme pp. 269-270.

Q.E.D.

Notice that in the "classical" Kolmogorov Extension Theorem the inverses system of measure spaces $\left(\left(\mathbb{R}^{n}, B\left(\mathbb{R}^{n}\right), \mu_{n}\right), \mathbb{N}, p r_{m n}\right)$ meets the condition of the above theorem.

\section{The general result}

The key notion of the paper:

Definition 6. The inverse system of measure spaces $\left(\left(X_{n}, \mathcal{M}_{n}, \mu_{n}\right), \mathbb{N}, f_{m n}\right)$ is $\varepsilon$ - complete, if $\forall \varepsilon \in[0,1], \forall(m \leq n)$, and $\forall A \subseteq X_{m}$ :

$$
\left(\mu_{n}^{*}\left(f_{m n}^{-1}(A)\right)=\varepsilon\right) \Rightarrow\left(\mu_{m}^{*}(A)=\varepsilon\right) .
$$

\footnotetext{
${ }^{1}$ Metivier's assumption is weaker, he requires that the measures should be non-negative $\sigma$-finite measures, and the index set is s.t. that has countable cofinal subset.
} 
It is a slight calculation to verify that Halmos' [6] example (exercise (3) pp. 214-215) is not an $\varepsilon$-complete inverse system of measure spaces. Furthermore, the reader may wonder whether it is enough to weaken $\varepsilon$-completeness for the case of $\varepsilon=0$. The answer is "not," if we manipulate Halmos' above mentioned example in such a way that the diagonal has $1-\varepsilon$ measure, and the set of the off diagonal elements has $\varepsilon$ (we distort Halmos' example by $\varepsilon)$, then we get at an 0 -complete inverse system of measure spaces having no inverse limit.

A further feature, the $\varepsilon$-completeness is not necessary condition for the existence of inverse limit. For this fact see the following inverse system of measure spaces:

$$
\left(\left(\left[0, \frac{1}{n+1}\right),\left\{\emptyset,\left[0, \frac{1}{n+1}\right)\right\}, \delta_{0}\right), \mathbb{N}, i d_{\left.0, \frac{1}{n+1}\right)}\right),
$$

where $\delta_{0}$ is for the Dirac measure concentrated at point 0 . It is easy to verify that (1) is an inverse system of measure spaces and its inverse limit is $\left(\{0\},\{\emptyset,\{0\}\}, \delta_{0}\right)$. However, (1) is not $\varepsilon$-complete.

The next theorem is the main result of this paper.

Theorem 7. Let $\left(\left(X_{n}, \mathcal{M}_{n}, \mu_{n}\right), \mathbb{N}, f_{m n}\right)$ be an $\varepsilon$-complete inverse system of measure spaces. Then $(X, \mathcal{M}, \mu) \stackrel{\circ}{=} \lim \left(\left(X_{n}, \mathcal{M}_{n}, \mu_{n}\right), \mathbb{N}, f_{m n}\right)$ exists and is unique.

Proof. (1) $X \stackrel{\circ}{=} \lim _{(}\left(X_{n}, \mathbb{N}, f_{m n}\right) \neq \emptyset: \forall n$ : let

$$
Q_{n} \stackrel{\circ}{=}\left\{x \in X_{0} \mid f_{0 n}^{-1}(\{x\})=\emptyset\right\} .
$$

It is clear that $\forall n: Q_{n} \subseteq Q_{n+1}$.

From $\varepsilon$-completeness $\forall n$ :

$$
\mu_{0}^{*}\left(Q_{n}\right)=0
$$

hence

$$
\mu_{0}^{*}\left(\bigcup_{n} Q_{n}\right)=0
$$

therefore

$$
\complement\left(\bigcup_{n} Q_{n}\right) \neq \emptyset
$$

Finally, from the definition of $\left\{Q_{n}\right\}_{n}, \forall x_{0} \in \complement\left(\bigcup_{n} Q_{n}\right): \exists x \in X$ such that $x_{0}=p_{0}(x)$, where $p_{0}: X \rightarrow X_{0}$ is the canonical projection. 
(2) $\bigcup_{n} p_{n}^{-1}\left(\mathcal{M}_{n}\right)$ is a field, and $\mu$ is an additive set function on it: From point (1) $\bigcup_{n} p_{n}^{-1}\left(\mathcal{M}_{n}\right)$ is a field.

$\forall A \in \bigcup_{n} p_{n}^{-1}\left(\mathcal{M}_{n}\right)$ : let

$$
\mu(A) \stackrel{\circ}{=} \mu_{n}(\hat{A}),
$$

where $n$ and $\hat{A} \in \mathcal{M}_{n}$ are such that $A=p_{n}^{-1}(\hat{A})$.

Let $\hat{A} \in \mathcal{M}_{m}$ and $\hat{B} \in \mathcal{M}_{m^{\prime}}$ be such that $p_{m}^{-1}(\hat{A})=p_{m^{\prime}}^{-1}(\hat{B})$. Then $\exists n^{*}$ : $n^{*} \geq m$ and $n^{*} \geq m^{\prime}$, and $\forall n \geq n^{*}$ : let

$$
Q_{n}^{\prime} \stackrel{\circ}{=}\left\{x \in X_{n^{*}} \mid f_{n^{*} n}^{-1}(\{x\})=\emptyset\right\} .
$$

It is clear that $\forall n \geq n^{*}: Q_{n}^{\prime} \subseteq Q_{n+1}^{\prime}$.

From $\varepsilon$-completeness $\forall n \geq n^{*}$ :

$$
\mu_{n^{*}}^{*}\left(Q_{n}^{\prime}\right)=0
$$

therefore

$$
\mu_{n^{*}}^{*}\left(\bigcup_{n \geq n^{*}} Q_{n}^{\prime}\right)=0
$$

$p_{m}^{-1}(\hat{A})=p_{m^{\prime}}^{-1}(\hat{B})$ implies that

$$
f_{m n^{*}}^{-1}(\hat{A}) \triangle f_{m^{\prime} n^{*}}^{-1}(\hat{B}) \subseteq \bigcup_{n \geq n^{*}} Q_{n}^{\prime}
$$

where $\triangle$ is for the symmetric difference. Put it differently $f_{n n^{*}}^{-1}(\hat{A}) \triangle f_{m^{\prime} n^{*}}^{-1}(\hat{B}) \in$ $\mathcal{M}_{n^{*}}$ and $\mu_{n^{*}}\left(f_{m n^{*}}^{-1}(\hat{A}) \triangle f_{m^{\prime} n^{*}}^{-1}(\hat{B})\right)=0$. Then

$$
\mu_{m}(\hat{A})=\mu_{m^{\prime}}(\hat{B})
$$

i.e. $\mu$ is well-defined (unique).

By repeating the above reasoning we get at that $\mu$ is additive.

(3) $\mu$ is $\sigma$-additive: Let $\left\{A_{n}\right\}_{n}, \forall n: A_{n} \in \mathcal{M}_{n}$ be such sets that $f_{n n+1}^{-1}\left(A_{n}\right) \supseteq$ $A_{n+1}$, and $\bigcap_{n} p_{n}^{-1}\left(A_{n}\right)=\emptyset$ (i.e. we show that $\mu$ is upper $\sigma$-continuous at $\emptyset$ ).

$\forall n$ : let

$$
L_{n} \stackrel{\circ}{=}\left\{x \in A_{0} \mid f_{0 n}^{-1}(\{x\}) \cap A_{n}=\emptyset\right\} .
$$

It is clear that $\forall n: L_{n} \subseteq L_{n+1}$.

Since $\forall n: f_{n n+1}^{-1}\left(A_{n}\right) \supseteq A_{n+1}$, therefore $\forall n$ : 


$$
f_{0 n}^{-1}\left(L_{n}\right) \subseteq f_{0 n}^{-1}\left(A_{0}\right) \backslash A_{n},
$$

hence

$$
\mu_{n}^{*}\left(f_{0 n}^{-1}\left(L_{n}\right)\right) \leq \mu_{0}\left(A_{0}\right)-\mu_{n}\left(A_{n}\right) .
$$

$\varepsilon$-completeness implies that $\forall n$ :

$$
\mu_{0}^{*}\left(L_{n}\right) \leq \mu_{0}\left(A_{0}\right)-\mu_{n}\left(A_{n}\right) .
$$

$\left\{\mu_{n}\left(A_{n}\right)\right\}_{n}$ is a bounded monotonic decreasing sequence of real numbers, hence it is convergent. $\mu_{0}$ is a probability measure, hence

$$
\mu_{0}^{*}\left(\bigcup_{n} L_{n}\right)=\lim _{n \rightarrow \infty} \mu_{0}^{*}\left(L_{n}\right) \leq \mu_{0}\left(A_{0}\right)-\lim _{n \rightarrow \infty} \mu_{n}\left(A_{n}\right) .
$$

From the definition of $\left\{A_{n}\right\}_{n}, \bigcap_{n} p_{n}^{-1}\left(A_{n}\right)=\emptyset$, hence $\lim _{n \rightarrow \infty} L_{n}=A_{0}$ : $\mu_{0}^{*}\left(\bigcup_{n} L_{n}\right)=\mu_{0}\left(A_{0}\right)$, moreover, from point $(2): \forall n: \mu\left(p_{n}^{-1}\left(A_{n}\right)\right) \stackrel{\circ}{=} \mu_{n}\left(A_{n}\right)$, hence

$$
\mu\left(p_{n}^{-1}\left(A_{n}\right)\right) \rightarrow 0
$$

(4) The extension of $\mu$ : From point (3) $\mu$ is a probability measure on the field $\bigcup_{n} p_{n}^{-1}\left(\mathcal{M}_{n}\right)$, hence it can be uniquely extended onto $\mathcal{M} \stackrel{\circ}{=} \sigma\left(\bigcup_{n} p_{n}^{-1}\left(\mathcal{M}_{n}\right)\right)$.

Q.E.D.

Remark 8. It is worth noticing that the conditions of Metivier's theorem (Theorem 5.) imply that the considered inverse system of measure spaces is $\varepsilon$-complete.

Let $n \in \mathbb{N}$ and $A \subseteq X_{n}$ be arbitrarily fixed, and $\varepsilon \stackrel{\circ}{=} \mu_{n+1}^{*}\left(f_{n n+1}^{-1}(A)\right)$. Since $\mathcal{M}_{n+1}$ is a $\sigma$-field, $\exists B \in \mathcal{M}$ s.t. $B \subseteq \complement f_{n n+1}^{-1}(A)$ and $\mu_{n+1}(B)=1-\varepsilon$.

From point 6. of Theorem 5. $\exists\left\{C_{m}\right\} \subseteq \mathcal{C}_{n+1}$ s.t. $\forall m: C_{m} \subseteq B$ and $\lim _{m \rightarrow \infty} \mu_{n+1}\left(C_{m}\right)=1-\varepsilon$.

From points 2. and 3. of Theorem 5. $\forall m: f_{n n+1}\left(C_{m}\right) \in \mathcal{M}_{n}$ and $\mu_{n}\left(f_{n n+1}\left(C_{m}\right)\right) \geq \mu_{n+1}\left(C_{m}\right)$.

Since $f_{n n+1}^{-1}(\complement A)=\complement f_{n n+1}^{-1}(A), \forall m: f_{n n+1}\left(C_{m}\right) \subseteq \complement A$, and $\lim _{m \rightarrow \infty} \mu_{n+1}\left(C_{m}\right)=$ $1-\varepsilon$ implies that $\mu^{*}(A)=\varepsilon$.

At first glance it seems that Theorem 7, requires much less than Theorem 5., but it provides the same result. From the viewpoint of the existence of a unique inverse limit of an inverse system of measure spaces it is true, however, genererally we can say only that while all the previous results require more 
(Theorem 5. is included) they also provide more, they state not only that the inverse limit exists and is unique, but they characterize it. On the other hand, the characterization is used in their proofs.

\section{An example}

First we introduce the measurable structure used in this section.

Definition 9. Let $(X, \mathcal{M})$ be arbitrarily fixed measurable space, and denote $\Delta(X, \mathcal{M})$ the set of the probability measures on it. Then the $\sigma$-field $\mathcal{A}^{*}$ on $\Delta(X, \mathcal{M})$ is defined as follows:

$$
\mathcal{A}^{*} \stackrel{\circ}{=}(\{\{\mu \in \Delta(X, \mathcal{M}) \mid \mu(A) \geq p\}, A \in \mathcal{M}, p \in[0,1]\}) .
$$

In other words

$$
\mathcal{A}^{*} \stackrel{\circ}{=} \sigma\left(\bigcup_{A \in \mathcal{M}} \operatorname{pr}_{A}^{-1}(B([0,1]))\right)
$$

where $\operatorname{pr}_{A}:\left.[0,1]^{\mathcal{M}}\right|_{\Delta(X, \mathcal{M})} \rightarrow[0,1]_{A},[0,1]_{A}$ is the $A$ copy of $[0,1]^{\mathcal{M}}$, and $B([0,1])$ is for the Borel $\sigma$-field on $[0,1]$.

The $\mathcal{A}^{*} \sigma$-field is specially interesting in interactive epistemology. Games with incomplete information are such games where the players are uncertain about some parameters of the game (the description of the game). In such games it is a cardinal problem that what the players believe about the game, and what the players believe about the other players' beliefs about the game an so on. Interactive epistemology is on this problem, it deals with the players' beliefs and knowledge. It is usual in the literature that the players' beliefs are modeled by probability measures.

In order to talk about the players' beliefs we need sentences like player $i$ believe with at least probability $\alpha$ that event $A$ happens (see e.g. Aumann [1], Heifetz and Samet [9]). This requirement is formalized in Definition 9. More precisely, the $\sigma$-field in Definition 9, is the weakest $\sigma$-field among those meet the above requirement, where the events (of the base space) are of $\mathcal{M}$.

Notice that $\mathcal{A}^{*}$ is not a fixed $\sigma$-field, it depends on the measurable space on which the probability measures are defined. Therefore $\mathcal{A}^{*}$ is similar to the weak* topology which depends on the topology of the base (primal) space. Henceforth the non-written $\sigma$-fields are the $\mathcal{A}^{*} \sigma$-fields.

The following diagram introduces the mathematical problem considered in this section. Altough Mertens et al.'s [13] consider topological spaces and Borel $\sigma$-fields, and compact regular measures, and our model is purely 
measurable the following formalization of the problem conceptually comes from Mertens et al.'s paper.

Definition 10. In diagram (2)

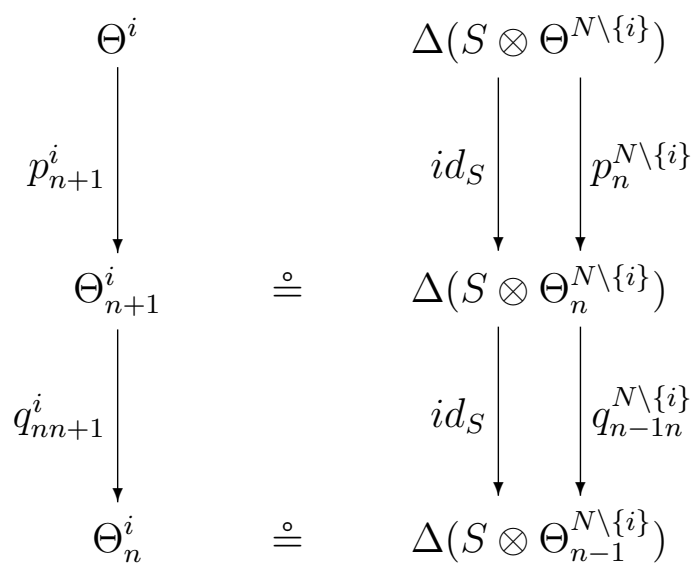

- $N$ is an arbitrary set,

- $i \in N$ is an arbitrarily element of $N$,

- $n \in \mathbb{N}$,

- $(S, \mathcal{A})$ is an arbitrary measurable space, moreover $\forall j \in N$ :

- $\# \Theta_{-1}^{j}=1$,

- $q_{-10}^{j}: \Theta_{0}^{j} \rightarrow \Theta_{-1}^{j}$,

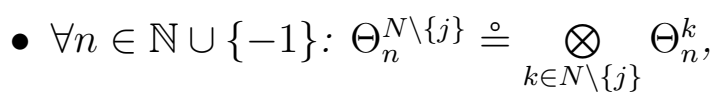

- $\forall m, n \in \mathbb{N}, m \leq n, \forall \mu \in \Theta_{n}^{j}$ :

$$
\left.q_{m n}^{j}(\mu) \stackrel{\circ}{=} \mu\right|_{S \otimes \Theta_{m-1}^{N \backslash\{j\}}},
$$

therefore $q_{m n}^{j}$ is a measurable mapping.

- $\Theta^{j} \stackrel{\circ}{=} \lim _{\longleftarrow}\left(\Theta_{n}^{j}, \mathbb{N} \cup\{-1\}, q_{m n}^{j}\right)$,

- $\forall n \in \mathbb{N} \cup\{-1\}: p_{n}^{j}: \Theta^{j} \rightarrow \Theta_{n}^{j}$ is canonical projection, 
- $\forall m, n \in \mathbb{N} \cup\{-1\}, m \leq n: q_{m n}^{N \backslash\{j\}}$ is the product of the mappings $q_{m n}^{k}$, $k \in N \backslash\{j\}$, and so is $p_{n}^{N \backslash\{j\}}$ of $p_{n}^{k}, k \in N \backslash\{j\}$, therefore both mappings are measurable,

- $\Theta^{N \backslash\{j\}} \stackrel{\circ}{=} \bigotimes_{k \in N \backslash\{j\}} \Theta^{k}$.

Let me give some intuitions what the above definition is about. Let $i$ be an arbitrary player and $(S, \mathcal{A})$ be the parameter space. The paramer space can be interpreted as the space that contains every parameter of the game (e.g. payoffs, etc.). Then $\Theta_{1}^{i}$ is the space of the so called first order beliefs of player $i$, i.e. this consists of player $i$ 's beliefs about the parameters of the game.

We assume that every player knows her own beliefs, so does player $i$, therefore it is enough to focus on the beliefs of players not $i$. Then $\Theta_{2}^{i}$ is the space of the so called second order beliefs of player $i$, i.e. this consists of player $i$ 's beliefs about the other players' beliefs about parameters of the game. In the same way we can interpret $\Theta_{n}^{i}$ as the space of player $i$ 's $n$th order beliefs. Finally $\Theta^{N}$ consists of every player's every hierarchy of beliefs i.e. of every player's arbitrary high order beliefs.

In this framework the goal is to "construct" an object, so called type space (see Heifetz and Samet), which consists of the parameters and all the hierarchies of all players, and for any player $i$, and for any probability measure $\mu$ on $S \otimes \Theta^{N \backslash\{i\}}$ there exists $\theta^{i} \in \Theta^{i}$ s.t.

$$
\left(S \otimes \Theta^{N \backslash\{i\}}, \mu\right)=\left(\left(S \otimes \Theta_{n}^{N \backslash\{i\}}, p_{n+1}^{i}\left(\theta^{i}\right)\right), \mathbb{N} \cup\{-1\},\left(i d_{S}, q_{m n}^{N \backslash\{i\}}\right)\right) .
$$

An object having the above property is an ideal one for the propurses of game theory.

The following proposition formalizes the above discussed goal. Since every previous result on this problem - Mertens and Zamir [12], Brandenburger and Dekel [4], Heifetz [8], Mertens at al., Pintér [17] among others - applies topological assumptions and ours do not, the next result is new.

Proposition 11. $\forall i \in N: \Theta^{i}=\Delta\left(S \otimes \Theta^{N \backslash\{i\}}\right)$, i.e. they are measurable isomorphic.

It is easy to verify that all we need to prove is that $\forall i \in N, \forall \theta^{i} \in \Theta^{i}$ : the inverse system of measure spaces

$$
\left(\left(S \otimes \Theta_{n}^{N \backslash\{i\}}, p_{n+1}^{i}\left(\theta^{i}\right)\right), \mathbb{N} \cup\{-1\},\left(i d_{S}, q_{m n}^{N \backslash\{i\}}\right)\right)
$$


admits a unique inverse limit, where $\left(i d_{S}, q_{m n}^{N \backslash\{i\}}\right)$ is the product of the given mappings.

In order to apply Theorem 7, we need the following result.

Lemma 12. Let $\left(X, \mathcal{M}_{1}\right)$ and $\left(X, \mathcal{M}_{2}\right)$ be such measurable spaces that $\mathcal{M}_{1} \subseteq$ $\mathcal{M}_{2}$, and $f: \Delta\left(X, \mathcal{M}_{2}\right) \rightarrow \Delta\left(X, \mathcal{M}_{1}\right)$ be defined as $\forall \nu \in \Delta\left(X, \mathcal{M}_{2}\right)$ :

$$
\left.f(\nu) \stackrel{\circ}{=}\right|_{\left(X, \mathcal{M}_{1}\right)} .
$$

Moreover, let $\mu_{2}$ be an arbitrary measure on $\Delta\left(X, \mathcal{M}_{2}\right)$ and $\left.\mu_{1} \stackrel{\circ}{=} \mu_{2}\right|_{\Delta\left(X, \mathcal{M}_{1}\right)}$. Then $\forall A \subseteq \Delta\left(X, \mathcal{M}_{1}\right), \forall \varepsilon \in[0,1]$ :

$$
\left(\mu_{2}^{*}\left(f^{-1}(A)\right)=\varepsilon\right) \Rightarrow\left(\mu_{1}^{*}(A)=\varepsilon\right) .
$$

Proof. Let $A \subseteq \Delta\left(X, \mathcal{M}_{1}\right)$ and $\varepsilon \in[0,1]$ be arbitrarily fixed. Then it is slight calculation to verify that

$$
f^{-1}(A)=\left(\bigcap_{B \in \mathcal{M}_{1}} \operatorname{pr}_{B}^{-1}(\{\nu(B) \mid \nu \in A\})\right) \cap\left(\bigcap_{B \in \mathcal{M}_{2} \backslash \mathcal{M}_{1}} \operatorname{pr}_{B}^{-1}\left(\left[\left(l_{B}, u_{B}\right)\right]\right)\right),
$$

where $l_{B} \stackrel{\circ}{=} \sup _{B \supseteq C \in \mathcal{M}_{1}} \inf _{\nu \in A} \nu(C), u_{B} \stackrel{\circ}{=} \inf _{B \subseteq D \in \mathcal{M}_{1}} \sup _{\nu \in A} \nu(D)$,

$$
\left[\left(l_{B}, u_{B}\right) \ominus= \begin{cases}\left(l_{B}, u_{B}\right), & \text { if } \forall \nu \in A, \forall C, D \in \mathcal{M}_{1}, C \subseteq B, B \subseteq D: \\ & \nu(C)<l_{B} \text { and } u_{B}<\nu(D) \\ {\left[l_{B}, u_{B}\right),} & \text { if } \exists \nu \in A, \exists C \in \mathcal{M}_{1}, C \subseteq B: l_{B}=\nu(C), \\ & \text { and } \forall \nu \in A, \forall D \in \mathcal{M}_{1}, B \subseteq D: u_{B}<\nu(D) \\ \left(l_{B}, u_{B}\right], & \text { if } \forall \nu \in A, \forall C \in \mathcal{M}_{1}, C \subseteq B: \nu(C)<l_{B}, \\ & \text { and } \exists \nu \in A, \exists D \in \mathcal{M}_{1}, B \subseteq D: u_{B}=\nu(D) \\ {\left[l_{B}, u_{B}\right],} & \text { if } \exists \nu, \nu^{\prime} \in A, \exists C, D \in \mathcal{M}_{1}, C \subseteq B, B \subseteq D: \\ & l_{B}=\nu(C) \text { and } u_{B}=\nu^{\prime}(D)\end{cases}\right.
$$

and $p r_{B}:\left.[0,1]^{\mathcal{M}_{2}}\right|_{\Delta\left(X, \mathcal{M}_{2}\right)} \rightarrow[0,1]_{B}$.

$\forall B \in \mathcal{M}_{2} \backslash \mathcal{M}_{1}$ : let $L_{B}, U_{B} \in \mathcal{M}_{1}$ be such that $L_{B} \subseteq B, B \subseteq U_{B}$, and $l_{B}=\inf _{\nu \in A} \nu\left(L_{B}\right), u_{B}=\sup _{\nu \in A} \nu\left(U_{B}\right)\left(\mathcal{M}_{1}\right.$ is a $\sigma$-field, so there are such sets $)$.

Then $\forall B \in \mathcal{M}_{2} \backslash \mathcal{M}_{1}$ :

$$
f^{-1}(A) \subseteq p r_{L_{B}}^{-1}\left(\left[\left(l_{B}, 1\right]\right) \cap p r_{U_{B}}^{-1}\left(\left[0, u_{B}\right)\right]\right) \subseteq p r_{B}^{-1}\left(\left[\left(l_{B}, u_{B}\right)\right]\right) .
$$

Put the above term differently, $\operatorname{pr}_{L_{B}}^{-1}\left(\left[\left(l_{B}, 1\right]\right) \cap \operatorname{pr}_{U_{B}}^{-1}\left(\left[0, u_{B}\right)\right]\right)$ is not worse in approximating $f^{-1}(A)$ via $B$ than $\operatorname{pr}_{B}^{-1}\left(\left[\left(l_{B}, u_{B}\right)\right]\right)$.

Therefore, from Definition 9, and equation (4): if $\mu_{2}^{*}\left(f^{-1}(A)\right)=\varepsilon$ then $\exists\left\{C_{n}\right\}_{n} \subseteq \mathcal{M}_{1}$ and $\exists\left\{D_{m}\right\}_{m} \subseteq \mathcal{M}_{2} \backslash \mathcal{M}_{1}$ : 


$$
f^{-1}(A) \subseteq\left(\bigcap_{n} p r_{C_{n}}^{-1}\left(B_{C_{n}}\right)\right) \cap\left(\bigcap_{m} p r_{D_{m}}^{-1}\left(\left[\left(l_{D_{m}}, u_{D_{m}}\right)\right]\right)\right),
$$

and

$$
\mu_{2}\left(\bigcap_{n} p r_{C_{n}}^{-1}\left(B_{C_{n}}\right)\right) \cap\left(\bigcap_{m} p r_{D_{m}}^{-1}\left(\left[\left(l_{D_{m}}, u_{D_{m}}\right)\right]\right)\right)=\varepsilon,
$$

where $B_{C_{n}} \in B([0,1])$ (Borel set of $[0,1]$ ).

(5) implies that $\exists\left\{K_{n}\right\}_{n} \subseteq \mathcal{M}_{1}$ :

$$
f^{-1}(A) \subseteq \bigcap_{n} p r_{K_{n}}^{-1}\left(B_{K_{n}}\right),
$$

$\left(B_{K_{n}} \in B([0,1])\right)$ and

$$
\mu_{2}\left(\bigcap_{n} p r_{K_{n}}^{-1}\left(B_{K_{n}}\right)\right)=\varepsilon
$$

i.e.

$$
A \subseteq \bigcap_{n} \operatorname{prr}_{K_{n}}^{-1}\left(B_{K_{n}}\right) \quad \text { and } \quad \mu_{1}\left(\bigcap_{n} \operatorname{prr}_{K_{n}}^{-1}\left(B_{K_{n}}\right)\right)=\varepsilon \text {, }
$$

where $\operatorname{prr}_{K_{n}}:\left.[0,1]^{\mathcal{M}_{1}}\right|_{\Delta\left(X, \mathcal{M}_{1}\right)} \rightarrow[0,1]_{K_{n}}$. Therefore $\mu_{2}^{*}\left(f^{-1}(A)\right)=\varepsilon$ implies that

$$
\mu_{1}^{*}(A)=\varepsilon
$$

Q.E.D.

The proof of Proposition 11. Lemma 12, $j \in$ and $n \in \mathbb{N}$ are arbitrarily fixed,

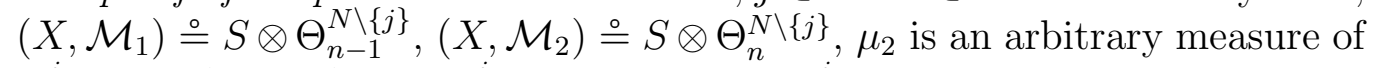
$\Theta_{n+1}^{j} \stackrel{\circ}{=} \Delta\left(X, \mathcal{M}_{2}\right), f \stackrel{\circ}{=} q_{n n+1}^{j}$. The case of $q_{-10}^{j}$ is trivial, finally if $\forall j \in N$ : $q_{n n+1}^{j}$ meets the given property then so does $\left(i d_{S}, q_{n n+1}^{N \backslash\{i\}}\right)$., implies that the inverse system of measure spaces (3) is $\varepsilon$-complete, so from Theorem 7 , it has a unique inverse limit, i.e. $\forall i \in N$ :

$$
\Theta^{i}=\Delta\left(S \otimes \Theta^{N \backslash\{i\}}\right)
$$

Q.E.D. 


\section{$5 \quad$ Final remarks}

We give two straightforward generalizations of Theorem 7. Henceforth we assume that $(I, \leq)$ is a right directed set.

A notion: Bochner [2] introduced the concept of sequential maximality, later Millington and Sion [15] "weakened" it, and got at the concept of almost sequential maximality.

Definition 13. The inverse system of measure spaces $\left(\left(X_{i}, \mathcal{M}_{i}, \mu_{i}\right),(I, \leq\right.$ ), $f_{i j}$ ) is almost sequentially maximal (a.s.m.) , if $\forall i_{1} \leq i_{2} \leq \ldots \in I$ chains $\exists A_{i_{n}} \subseteq X_{i_{n}}$ :

- $\forall(n \leq m): f_{i_{n} i_{m}}^{-1}\left(A_{i_{n}}\right) \subseteq A_{i_{m}}$

- $\forall n: \mu_{i_{n}}^{*}\left(A_{i_{n}}\right)=0$,

- $\left(\forall n: x_{i_{n}} \in\left(X_{i_{n}} \backslash A_{i_{n}}\right)\right.$ and $\left.x_{i_{n}}=f_{i_{n} i_{n+1}}\left(x_{i_{n+1}}\right)\right) \Rightarrow\left(\exists x \in \lim \left(X_{i},(I\right.\right.$, $\left.\leq), f_{i j}\right)$ such that $\left.\forall n: x_{i_{n}}=p_{i_{n}}(x)\right)$.

The first generalization:

Theorem 14. Let $\left(\left(X_{i}, \mathcal{M}_{i}, \mu_{i}\right),(I, \leq), f_{i j}\right)$ be such an inverse system of measure spaces that is

1. almost sequentially maximal,

2. $\varepsilon$-complete.

Then $\lim _{(}\left(\left(X_{i}, \mathcal{M}_{i}, \mu_{i}\right),(I, \leq), f_{i j}\right)$ exists and unique.

An other generalization:

Theorem 15. Let $\left(\left(X_{i}, \mathcal{M}_{i}, \mu_{i}\right),(I, \leq), f_{i j}\right)$ be such an inverse system of measure spaces that (is)

1. $(I, \leq)$ has countable cofinal subset,

2. $\varepsilon$-complete.

Then $\lim _{(}\left(\left(X_{i}, \mathcal{M}_{i}, \mu_{i}\right),(I, \leq), f_{i j}\right)$ exists and unique.

The two above theorems are direct corollaries of Theorem 7 . 


\section{References}

[1] Aumann, R.J.: "Interactive epistemology II., Probability" International Journal of Game Theory 28, 301-314. (1999)

[2] Bochner S.: Harmonic Analysis and the Theory of Probability, University of California Press (1955)

[3] Bourbaki, N.: Éléments de Mathématque, Intégration, Livre VI, Chapitre IX, Intégration sur Les Espaces Topologiques Séparés, Hermann (1969)

[4] Brandenburger, A., E. Dekel: "Hierarchies of beliefs and common knowledge" Journal of Economic Theory 59, 189-198. (1993)

[5] Choksi, J. R.: "Inverse limits of measure spaces" Proc. London Math. Soc. 8(Ser 3), 321-342. (1958)

[6] Halmos, P. R.: Measure Theory, Springer-Verlag (1974)

[7] Harsányi, J.: "Games with incomplete information played by bayesian players part I., II., III." Management Science 14, 159-182., 320-334., 486-502. (1967-1968)

[8] Heifetz, A.: "The bayesian formulation of incomplete information - the non-compact case" International Journal of Game Theory 21, 329-338. (1993)

[9] Heifetz, A., D. Samet: "Topology-free typology of beliefs" Journal of Economic Theory 82, 324-341. (1998)

[10] Kolmogorov, A. N.: Foundations of the Theory of Probability, Second English Edition, translation edited by Nathan Morrison, Chelsea Publishing Company (1956)

[11] Mallory, D. J., M Sion.: "Limits of inverse systems of measures" Ann. Inst. Fourier 21, 25-57. (1971)

[12] Mertens, J. F., S. Zamir: "Formulations of bayesian analysis for games with incomplete informations" International Journal of Game Theory 14, 1-29. (1985)

[13] Mertens, J. F., S. Sorin, S. Zamir: "Repeated games part A" CORE Discussion Paper No. 9420 (1994) 
[14] Metivier, M.: "Limites projectives de measures, martingales, applications" Annali di Matematica 63, 225-352. (1963)

[15] Millington, H., M. Sion: "Inverse systems of group-valued measures" Pacific Journal of Mathematics 44, 637-650. (1973)

[16] v. Neumann J., Morgenstern O.: Theory of Games and Economic Behavior, Princeton University Press, (1953)

[17] Pintér, M.: "Type space on a purely measurable parameter space" Economic Theory 26, 1239-139. (2005)

[18] Rao, M. M.: Stochastic Processes and Integration, Sijthoff \& Noordhoof (1979) 